\title{
FITOHORMONAS REDUCEN DAÑOS POR Nacobbus aberrans EN TOMATE (Solanum lycopersicum L.)
}

\section{PHYTOHORMONES REDUCE DAMAGE BY Nacobbus aberrans IN TOMATO (Solanum lycopersicum L.)}

Susana B. Martínez ${ }^{1 *}$, Mariana Garbi ${ }^{1}$, Lucrecia Puig ${ }^{1}$, Guillermo B. Cap², Daniel O. Gimenez ${ }^{3}$

${ }^{1}$ Facultad de Ciencias Agrarias y Forestales (FCAyF), Universidad Nacional de La Plata (UNLP). Calle 60 s/n, 1900 La Plata, Buenos Aires, Argentina.

${ }^{2}$ Instituto Nacional de Tecnología Agropecuaria (INTA), Chacra Experimental Gorina, Calles 501 y 149 , 1896 Joaquín Gorina, Buenos Aires, Argentina.

${ }^{3}$ Instituto de Fisiología Vegetal, UNLP, FCAyF-Facultad de Ciencias Naturales y Museo-Consejo Nacional de Investigaciones Científicas y Técnicas (CONICET), Diagonal 113 s/n, 1900 La Plata, Buenos Aires, Argentina.

* Autor para correspondencia E-mail: smarti@agro.unlp.edu.ar

Susana Martinez https://orcid.org/0000-0002-4256-7406

Mariana Garbi: ORCID: 0000-0002-0263-1901

\section{RESUMEN}

Existen antecedentes de la reducción de daños por nemátodos con el uso de elicitores, compuestos que estimulan defensas en las plantas, promoviendo el metabolismo secundario. Este trabajo tuvo como objetivos evaluar la nematofauna presente en el suelo y raíces del cultivo antecesor, y medir el efecto sobre el rendimiento y daño en raíces por Nacobbus aberrans en tomate (Solanum lycopersicum) ${ }^{\prime}$ Elpida', tratado por drench 24 horas previas al trasplante con $1 \mathrm{~mL}$ de ácido salicílico $0,50 \times 10^{-4} \mathrm{M}$ y $1,00 \times 10^{-4} \mathrm{M}$, etileno $0,35 \times 10^{-3} \mathrm{M}$ y $0,70 \times 10^{-3} \mathrm{M}$, ácido jasmónico $1,00 \times 10^{-4} \mathrm{M}$ y 1,00 $\times 10^{-5} \mathrm{M}$, dejando testigos sin tratar. En el suelo se identificaron nemátodos saprófitos, omnívoros y predatores, Helicotylenchus spp. y N. aberrans. El cultivo antecesor fue tomate 'Elpida' sin injertar e injertado sobre Maxifort, 9184, Multifort y Beaufort, identificándose huevos y N. aberrans J2 en todas las raíces, en cantidad significativamente menor en Maxifort y 9184. Las fitohormonas incrementaron la producción de frutos con peso mayor a $150 \mathrm{~g}$ y el rendimiento total respecto al testigo, produciendo menor número de agallas, índice de agallamiento y reproducción. Ácido salicílico $1,00 \times 10^{-4} \mathrm{M}$ produjo menor daño en raíces y mayor producción total y por racimos, seguido por etileno $0,70 \times 10^{-3}$ M y ácido jasmónico 1,00 × $10^{-5} \mathrm{M}$. Es importante continuar investigando aspectos prácticos del uso de elicitores y profundizar en la comprensión de su acción para el control de $N$. aberrans.

Palabras clave: elicitores, resistencia sistémica, ácido salicílico, etileno, ácido jasmónico.

\section{ABSTRACT}

There is evidence that indicates that elicitors reduce damage caused by nematodes. Elicitors are compounds that stimulate plant defense promoting secondary metabolism. The aims of this study were to evaluate the soil and previous root crop nematofauna in tomato 'Elpida', and determine the effect on yield and root damage by Nacobbus aberrrans. Soil drench applications were conducted 24 hours pre-transplanting with $1 \mathrm{~mL}$ of: salicilic acid $0.50 \times 10^{-4} \mathrm{M}$ and $1.00 \times 10^{-4} \mathrm{M}$, ethylen $0.35 \times 10^{-3} \mathrm{M}$

Recibido: 12 agosto 2020. Aceptado: 21 diciembre 2020. 
and $0.70 \times 10^{-3} \mathrm{M}$, jasmonic acid $1.00 \times 10^{-4} \mathrm{M}$ and $1.00 \times 10^{-5} \mathrm{M}$, including untreated plants as control. In the soil, saprophytic, omnivorous and predatory nematodes, as well as Helicotylenchus spp. and $N$. aberrans, were identified. The previous crop was ungrafted 'Elpida' plants and also grafted on Maxifort, 9184, Multifort and Beaufort. Eggs and N. aberrans J2 were identified in all roots, with significantly lower amounts in Maxifort and 9184. Phytohormones increased the production of fruits with weight higher than $150 \mathrm{~g}$ and total yield compared to the control, producing lower galls, galling and reproductive indexes. Salicylic acid $1.00 \times 10^{-4} \mathrm{M}$ produced lower damage to the roots and a higher total yield and truss yield, followed by ethylene $0.70 \times 10^{-3} \mathrm{M}$ and jasmonic acid $1.00 \times 10^{-5} \mathrm{M}$ treatments. Further research is required to provide deeper knowledge of the practical implications of using elicitors and enhance the understanding of their action for N. aberrans control.

Key words: elicitors, systemic resistance, salicylic acid, ethylene, jasmonic acid

\section{INTRODUCCIÓN}

El falso nemátodo del nudo de la raíz Nacobbus aberrans [(Thorne 1935) Thorne y Allen), 1944] (Nematoda, Pratylenchidae) presenta una amplia gama de hospedantes y distribución geográfica, además de gran capacidad reproductiva y elevado potencial para causar pérdidas de rendimiento; por lo que en numerosos países se han establecido medidas cuarentenarias para esta plaga (Méndez-Santiago, 2020).

Entre las alternativas para reducir los daños producidos por nemátodos se ha probado el tratamiento de las plantas con fitohormonas que actúan como elicitoras de resistencia sistémica. Los elicitores son compuestos que estimulan el sistema de defensa de la planta, promoviendo el metabolismo secundario, protegiendo de esta manera a las células y a la planta en su totalidad (Ramirez-Estrada et al., 2016). Este tipo de resistencia inducida, en general, no impide en forma inmediata la aparición de la enfermedad o daño, como lo harían los fitosanitarios utilizados tradicionalmente, pero reducen la severidad y su efecto es más duradero en el tiempo (Peteira, 2020).

La aplicación exógena de ácido jasmónico en distintas especies vegetales estimuló la resistencia directa a diversos insectos, a través de la reducción de su fecundidad, crecimiento y supervivencia (Laredo et al., 2017). Sánchez et al. (2010) indicaron que el ácido salicílico induce la expresión de genes de resistencia contra virus, bacterias y hongos patógenos; mientras que Suazo Castro (2019) observó una reducción en el número de mosca blanca (Trialeurodes vaporariorum) en tomate tratado con ácido abscísico y ácido salicílico, aplicados individualmente.

Nemátodos fitopatógenos como Globodera spp., Meloidogyne spp. y Heterodera spp., entre otros, actúan a través de mecanismos que suprimen la ruta del ácido salicílico, molécula señal para el proceso de resistencia sistémica adquirida, por lo que su aplicación en diversas especies ha servido para reducir la infestación (Samaniego-
Gámez et al., 2017; Gheysen y Mitchum, 2019). Por su parte, el etileno es un regulador importante frente a una amplia gama de estreses generados por heridas o ataque de patógenos a la planta, y al igual que el ácido jasmónico, actúa en la señalización para procesos de resistencia sistémica inducida (Samaniego-Gámez et al., 2017). Estudios desarrollados en Arabidopsis thaliana (L.) indicaron que interferencias en la ruta del etileno aumentaban las probabilidad de un rápido establecimiento de Heterodera schachtii (Marhavý, 2019); por otra parte, el rol del ácido jasmónico en la defensa de las plantas frente al ataque de nemátodos es menos conocido y ha presentado resultados más contradictorios (Gheysen y Mitchum, 2019). Sin embargo, se ha observado que su aplicación exógena fue efectiva para reducir la cantidad de huevos en las raíces de tomate, cuando las plantas fueron sometidas a la infestación posterior al tratamiento con Meloidogyne incognita (Zhou et al., 2015).

La mayor parte de los estudios sobre la reacción defensiva de las plantas al ataque de nemátodos, o el uso de elicitores para la reducción de los daños que provocan, se han focalizado en nemátodos de géneros distintos a Nacobbus spp. Sin embargo, en pimiento (Capsicum annum L.) se observó que $N$. aberrans indujo una reducción en el contenido total de fenoles solubles, la actividad de la peroxidasa y la L-fenilalaninaamoniaco-liasa, enzima clave en la ruta del fenilpropanoide, cuyas ramas llevan a la síntesis de compuestos con diversas funciones en las plantas, entre ellas las relacionadas a los mecanismos de defensa (López-Martínez et al., 2010). De esta manera, la utilización de fitohormonas con funciones elicitoras podría ser una alternativa potencialmente útil para mitigar los problemas causados por este nemátodo en el cultivo de tomate.

Este estudio tuvo como objetivo evaluar el efecto de la aplicación exógena de distintas dosis de ácido salicílico, ácido jasmónico y etileno, sobre el rendimiento y daño producido por Naccobus aberrans en las raíces de un cultivo de tomate conducido en invernadero, previa caracterización 
y cuantificación de la nematofauna presente en el sitio de ensayo.

\section{MATERIALES Y MÉTODOS}

El ensayo se condujo en un invernadero metálico de $24 \times 40 \mathrm{~m}$ con techo parabólico, ubicado en la Estación Experimental Julio Hirschhorn, perteneciente a la Facultad de Ciencias Agrarias y Forestales de la Universidad Nacional de La Plata, La Plata, Buenos Aires, Argentina (335' 42,6 " S; 60³3'35,6" W). Como cultivo antecesor a este ensayo se encontraba implantado tomate 'Elpida' sin injertar, e injertado sobre los portainjertos Maxifort F1, Beaufort F1, Multifort F1, y 9184. Antes de iniciar el nuevo ciclo de cultivo, se realizaron análisis de suelo y raíces para caracterizar y contabilizar la nematofauna presente. Los muestreos de raíces se realizaron a la finalización del cultivo antecesor, en mayo de 2013. En el suelo se tomaron muestras compuestas de $250 \mathrm{~g}$ en 21 puntos del invernadero, formadas por 5 muestras simples tomadas a $20 \mathrm{~cm}$ de profundidad en zig-zag. Se utilizó un muestreador formado por un tubo de $2,5 \mathrm{~cm}$ de diámetro por $20 \mathrm{~cm}$ de largo. En raíces se tomaron muestras cada tres plantas por parcela. Para la extracción de nemátodos del suelo se aplicó el método de centrifugación-flotación descripto por Hooper et al. (2005). Para la extracción a partir de raíces se utilizó el método de maceración descripto por Coyne et al. (2007). A partir de las extracciones, por observación microscópica, se contabilizó la cantidad de huevos y nemátodos, y se los identificó morfológicamente a nivel de género, en base a la morfología del aparato bucal y esófago. Se utilizó un diseño enteramente aleatorizado con 21 repeticiones. Los datos se sometieron a la prueba no paramétrica de Kruskal-Wallis, utilizando el programa Infostat (Di Rienzo et al., 2013).

El cultivo siguiente se trasplantó el 16 de enero de 2014, utilizando plantines de tomate 'Elpida' con 4 hojas verdaderas, que 24 horas antes fueron tratados con hormonas vegetales aplicadas por mojado del sustrato (drench) a razón de $1 \mathrm{~mL}$ por planta, según los siguientes tratamientos: 1) Ácido salicílico $0,5 \times 10^{-4} \mathrm{M}$; 2) Ácido salicílico $1,0 \times 10^{-4} \mathrm{M}$; 3) Etileno $0,35 \times 10^{-3} \mathrm{M}$; 4) Etileno $0,70 \times 10^{-3} \mathrm{M}$; 5) Ácido jasmónico $1,00 \times 10^{-4} \mathrm{M}$; 6) Ácido jasmónico $1,00 \times 10^{-5} \mathrm{M}$; 7), y Testigo, sin aplicación de hormonas, tratados con igual volumen de agua. La plantación fue en líneas con una distancia de $0,80 \mathrm{~m}$ entre lomos y $0,25 \mathrm{~m}$ entre plantas, sobre suelo cubierto con mulching negro, y riego por goteo, manteniendo el suelo a capacidad de campo. Las plantas se condujeron en forma vertical con hilo.
El ensayo se realizó según un diseño estadístico en bloques completos aleatorizados con 4 repeticiones, con 10 plantas por parcela. Se cosechó hasta el sexto racimo, registrando para cada racimo el rendimiento total y por categorías comerciales, definidas según el peso de fruto: primera, con frutos de peso igual o mayor a 150 $\mathrm{g}$; segunda, con frutos de $100 \mathrm{a} 149 \mathrm{~g}$, y tercera, con frutos de peso inferior a $100 \mathrm{~g}$. A partir de esos datos se calculó el rendimiento total y por categorías comerciales para el ciclo completo. Al finalizar el ensayo se extrajeron tres raíces por unidad experimental, se lavaron con agua potable, se secaron con papel absorbente y se registró el número de agallas, por conteo directo de agallas identificadas visualmente, y peso fresco de las raíces, por pesaje en balanza electrónica digital con precisión de $1 \mathrm{~g}$. Sobre 5 muestras de raíces se procedió a la extracción de huevos de nemátodos, aplicando la técnica descripta por Veremis et al. (1997). A partir de los datos previos se calculó: el índice de agallamiento, como el cociente entre el número de agallas y el peso fresco de raíces (Pérez-Rodríguez, 2008; Pérez-Rodríguez et al., 2011), clasificándolas según la escala propuesta por Taylor y Sasser (1978), donde $0=0$ agallas; $1=1-2$ agallas; $2=3-10$ agallas; $3=11-30$ agallas; $4=31-100$ agallas; $5=>100$ agallas; y el índice reproductivo, a través de la siguiente fórmula: Índice de Reproducción $=(\mathrm{P}$ var $\times 100) / \mathrm{P}$ testigo, donde $\mathrm{P}$ var es el número de huevos-J2 y $\mathrm{P}$ testigo el número de nematodos presentes en el suelo previo al trasplante.

Los datos de rendimiento fueron transformados por $\log (\mathrm{y}+1)$ previo a ser sometidos a análisis de la varianza. Las diferencias entre medias se compararon por la prueba de Tukey. Los análisis estadísticos se realizaron con el programa Infostat (Di Rienzo et al., 2013).

\section{RESULTADOS Y DISCUSIÓN}

Al momento del inicio del ensayo, el suelo se encontraba naturalmente infestado por $N$. aberrans, identificándose la presencia de juveniles J3 y J4. En magnitudes equivalentes se encontraron Helicotylenchus spp. y nemátodos saprófitos (Rhabditis spp. y Cephalobus spp.), así como nemátodos omnívoros o predatores como Mononchus spp., en número significativamente mayor que los citados previamente, y Dorylaimus spp., cuya presencia fue significativamente más alta que la de saprófitos y Helicotylenchus spp. (Tabla 1). La variedad de nemátodos identificados en el suelo de ensayo es coincidente con la reportada para otros sitios sometidos a distintas formas de manejo en el área hortícola de La Plata, destacándose la alta proporción de predatores 
Tabla 1. Caracterización y recuento de nemátodos presentes en el suelo del invernadero previo a trasplante de tomate 'Elpida' tratado con distintas dosis de fitohormonas.

Table 1. Characterization and counting of nematodes present in the greenhouse soil prior to transplantimg 'Elpida' tomato treated with different doses of phytohormones.

\begin{tabular}{lc}
\hline Género de nemátodos & Número en $\mathbf{1 0 0} \mathbf{~ c m}^{\mathbf{3}}$ de suelo \\
\hline Saprófitos (Rabditis spp., & $33 \mathrm{a}$ \\
Cephalobus spp.) & \\
Helicotylenchus spp. & $45 \mathrm{a}$ \\
Nacobbus aberrans J3 y J4 & $48 \mathrm{ab}$ \\
Dorylaimus spp. & $64 \mathrm{bc}$ \\
Mononchus spp. & $76 \mathrm{c}$ \\
\hline
\end{tabular}

Letras distintas indican diferencias significativas $(p<0,05)$

u omnívoros, en relación a los encontrados en esos lugares (Salas et al., 2015). La cantidad de omnívoros y predadores presentes en el invernadero de ensayo, en relación a otros tipos de nemátodos, podría considerarse un indicador de las buenas condiciones edáficas, dada su alta sensibilidad a disturbios del suelo, lo que permitiría una regulación de la multiplicación de los fitófagos (Azpilicueta et al., 2011). En el cultivo antecesor al de ensayo, $N$. aberrans se reprodujo tanto en las raíces de las plantas de tomate 'Elpida' sin injertar como en las injertadas, observándose un comportamiento diferencial entre los portainjertos, con una cantidad significativamente inferior de huevos y juveniles J2 en las raíces de Maxifort y 9184 (Tabla 2). La capacidad de este nemátodo para multiplicarse en portainjertos con las características de los utilizadas en este trabajo, con resistencia genética a Meloidogyne spp., es coincidente con lo reportado por Mitidieri et al. (2011), quienes observaron la tolerancia de distintos portainjertos a la presencia de $N$. aberrans, con un agallamiento significativamente menor que en las plantas sin injertar y un buen comportamiento de la combinación Elpida-Maxifort.

Los estadios J3 y J4 de N. aberrans, así como trozos de raíces de plantas infestadas han demostradoserinfectivosinduciendola formación de agallas en raíces de tomate, conservando esta capacidad por más de un año, aun cuando se los sometió a condiciones extremas de temperatura, humedad o ausencia de hospedantes susceptibles (Cristóbal et al., 2001), pudiendo considerarse que en las condiciones de ensayo proveyeron inóculo natural suficiente para la segunda parte del trabajo.

Los tratamientos con hormonas vegetales incrementaron significativamente el rendimiento en frutos de primera categoría y la producción total. La aplicación de ácido salicílico en su mayor concentración $\left(1,00 \times 10^{-4} \mathrm{M}\right)$ produjo aumentos significativos en ambas categorías, con una respuesta estadísticamente equivalente producida por el ácido jasmónico en dosis $1,00 \times 10^{-5} \mathrm{M}$, y por el etileno en dosis de $0,70 \times 10^{-3} \mathrm{M}$, en frutos de primera. Las plantas sin aplicación de hormonas presentaron la menor producción en frutos de primera, diferenciándose significativamente del resto de los tratamientos, con una producción total también significativamente más baja, con diferencias respecto a las plantas tratadas, a excepción de aquellas a las que se les aplicó ácido jasmónico en dosis de $1,00 \times 10^{-4} \mathrm{M}$. No se observaron efectos de la aplicación de hormonas en la producción de frutos de segunda y tercera categoría (Tabla 3). Las respuestas observadas para el total de la producción se dieron también en la cosecha por racimo, siendo más evidentes en el primer racimo, aunque el efecto del ácido salicílico $1,00 \times 10^{-4} \mathrm{M}$ se manifestó también en la producción de frutos de primera y rendimiento total del quinto y sexto racimo, particularmente respecto al testigo (Tablas 4 a 9). La respuesta de la producción de frutos a este tratamiento y su efecto sobre el primer racimo es coincidente con lo reportado por Yildirim y Dursun (2009) quienes registraron un rendimiento temprano significativamente más elevado en tomate asperjado por vía foliar con ácido salicílico 0,50 $\mathrm{mM}$.

Es sabido que las hormonas vegetales son utilizadas para mejorar el crecimiento, rendimiento y calidad de los productos cosechados, por su participación en diversos procesos fisiológicos (Avanci et al., 2010; TucuchHass et al., 2017). En pimiento (Capsicum annum L.) la aplicación de ácidos salicílico 0,1 y 0,2 mM a través de la solución nutritiva luego del trasplante produjo incrementos en la biomasa aérea y radical, producción de frutos y actividad fotosintética (Sánchez-Chávez et al., 2011), obteniéndose una respuesta equivalente en la misma especie por la doble aspersión (1 y 3 semanas después del 
Tabla 2. Recuento de huevos y juveniles J2 de $N$. aberrans (número g de raíz) en el cultivo antecesor al trasplante de tomate 'Elpida' tratado con distintas dosis de fitohormonas.

Table 2. Count of eggs and J2 juveniles of $N$. aberrans (root $g$ number) in the crop prior to the transplant of ${ }^{\prime}$ Elpida' tomato treated with different doses of phytohormones.

\begin{tabular}{lcc}
\hline Cultivo antecesor & Huevos & J2 \\
\hline Elpida-Maxifort & $20 \mathrm{a}$ & $24 \mathrm{a}$ \\
Elpida-9184 & $20 \mathrm{a}$ & $25 \mathrm{a}$ \\
Elpida-Beaufort & $36 \mathrm{~b}$ & $38 \mathrm{~b}$ \\
Elpida-Multifort & $41 \mathrm{~b}$ & $40 \mathrm{~b}$ \\
Elpida & $35 \mathrm{~b}$ & $38 \mathrm{~b}$ \\
\hline
\end{tabular}

Letras distintas en la columna indican diferencias significativas $(p<0,05)$

Tabla 3. Rendimiento total y por categorías comerciales $\left(\mathrm{kg} \mathrm{m}^{-2}\right)$ en tomate 'Elpida' tratado al trasplante con diferentes hormonas vegetales y dosis, cultivado en suelo infestado naturalmente con N. aberrans.

Table 3. Total and grade yield $\left(\mathrm{kg} \mathrm{m}^{-2}\right)$ of 'Elpida' tomato treated at transplant with different phytohormones and doses, grown in soil with natural infestation of $N$. aberrans.

\begin{tabular}{lcccc}
\hline \multirow{2}{*}{\multicolumn{1}{c}{ Tratamientos }} & \multicolumn{3}{c}{ Categorías comerciales } & \multirow{2}{*}{ Total } \\
\cline { 2 - 4 } & Primera & Segunda & Tercera & \\
\hline Ácido salicílico $0,5 \times 10^{-4} \mathrm{M}$ & $7,44 \mathrm{~b}$ & $3,71 \mathrm{a}$ & $0,76 \mathrm{a}$ & $11,91 \mathrm{bc}$ \\
Ácido salicílico $1,0 \times 10^{-4} \mathrm{M}$ & $15,45 \mathrm{c}$ & $5,15 \mathrm{a}$ & $1,05 \mathrm{a}$ & $21,66 \mathrm{~d}$ \\
Etileno $0,35 \times 10^{-3} \mathrm{M}$ & $8,84 \mathrm{~b}$ & $3,64 \mathrm{a}$ & $1,52 \mathrm{a}$ & $14,00 \mathrm{bc}$ \\
Etileno $0,70 \times 10^{-3} \mathrm{M}$ & $10,36 \mathrm{bc}$ & $3,03 \mathrm{a}$ & $0,96 \mathrm{a}$ & $14,35 \mathrm{bc}$ \\
Ácido jasmónico $1,00 \times 10^{-4} \mathrm{M}$ & $7,49 \mathrm{~b}$ & $2,63 \mathrm{a}$ & $1,04 \mathrm{a}$ & $11,16 \mathrm{ab}$ \\
Ácido jasmónico $1,00 \times 10^{-5} \mathrm{M}$ & $11,79 \mathrm{bc}$ & $3,24 \mathrm{a}$ & $0,91 \mathrm{a}$ & $15,65 \mathrm{~cd}$ \\
Testigo & $4,08 \mathrm{a}$ & $2,32 \mathrm{a}$ & $1,31 \mathrm{a}$ & $7,71 \mathrm{a}$ \\
$\mathrm{CV}$ & 2,64 & 4,45 & 7,89 & 1,71 \\
$\mathrm{R}^{2}$ & 0,83 & 0,49 & 0,37 & 0,87 \\
$\mathrm{p}$ & $<0,0001$ & 0,1343 & 0,4244 & $<0,0001$ \\
\hline
\end{tabular}

Letras distintas en la columna indican diferencias significativas, prueba de Tukey $(p<0,05)$

Tabla 4. Primer racimo: rendimiento total y por categorías comerciales $\left(\mathrm{kg} \mathrm{m}^{-2}\right)$ en tomate 'Elpida' tratado al trasplante con diferentes hormonas vegetales y dosis, cultivado en suelo infestado naturalmente con $N$. aberrans.

Table 4. First fruit truss: total and grade yield $\left(\mathrm{kg} \mathrm{m}^{-2}\right)$ of 'Elpida' tomato treated at transplant with different phytohormones and doses, grown in soil with natural infestation of $N$. aberrans.

\begin{tabular}{lcccl}
\hline \multirow{2}{*}{\multicolumn{1}{c}{ Tratamientos }} & \multicolumn{3}{c}{ Categorías comerciales } & \multirow{2}{*}{ Total } \\
\cline { 2 - 4 } & Primera & Segunda & Tercera & \\
\hline Ácido salicílico $0,5 \times 10^{-4} \mathrm{M}$ & $1,86 \mathrm{a}$ & $2,05 \mathrm{a}$ & $0,30 \mathrm{a}$ & $4,11 \mathrm{ab}$ \\
Ácido salićlico $1,0 \times 10^{-4} \mathrm{M}$ & $4,84 \mathrm{~d}$ & $2,28 \mathrm{a}$ & $0,28 \mathrm{a}$ & $7,41 \mathrm{~d}$ \\
Etileno $0,35 \times 10^{-3} \mathrm{M}$ & $2,88 \mathrm{bc}$ & $2,03 \mathrm{a}$ & $0,72 \mathrm{a}$ & $5,63 \mathrm{bcd}$ \\
Etileno $0,70 \times 10^{-3} \mathrm{M}$ & $2,98 \mathrm{bc}$ & $1,16 \mathrm{a}$ & $0,32 \mathrm{a}$ & $4,46 \mathrm{abc}$ \\
Ácido jasmónico $1,00 \times 10^{-4} \mathrm{M}$ & $3,31 \mathrm{bcd}$ & $1,26 \mathrm{a}$ & $0,44 \mathrm{a}$ & $5,00 \mathrm{abcd}$ \\
Ácido jasmónico $1,00 \times 10^{-5} \mathrm{M}$ & $4,27 \mathrm{~cd}$ & $2,19 \mathrm{a}$ & $0,32 \mathrm{a}$ & $6,78 \mathrm{~cd}$ \\
Testigo & $1,19 \mathrm{a}$ & $1,15 \mathrm{a}$ & $0,48 \mathrm{a}$ & $2,81 \mathrm{a}$ \\
$\mathrm{CV}$ & 3,88 & 6,44 & 14,48 & 3,04 \\
$\mathrm{R}^{2}$ & 0,81 & 0,49 & 0,24 & 0,77 \\
$\mathrm{p}$ & 0,0001 & 0,1483 & 0,6587 & 0,0007 \\
\hline
\end{tabular}

Letras distintas en la columna indican diferencias significativas, prueba de Tukey $(p<0,05)$ 
trasplante) de ácido jasmónico 0,5 mM (Awang et al., 2015). También en maíz (Zea mays) la aspersión foliar de plantas de 7 días de edad con ácido salicílico 0,1 y $1 \mu \mathrm{M}$ promovió incrementos significativos en componentes del rendimiento, como ancho y largo de la mazorca (Tucuch-Hass et al., 2017). Sin embargo, en las condiciones de este ensayo, además de la acción fisiológica de las hormonas, puede considerarse su efecto como elicitoras de resistencia a $N$. aberrans, conforme se verificó en la reducción significativa del número de agallas, índice de agallamiento y de reproducción registrado en las plantas tratadas. El ácido salicílico en la concentración de 1,00 x $10^{-4} \mathrm{M}$ se destacó en este sentido, promoviendo también aumento significativo del peso fresco de la raíz, con la presencia de menor número de agallas en relación al resto de los tratamientos, y nulos índices de agallamiento y reproducción (Tabla 10). Si bien, al igual que en la observación realizada por Molinari (2008), la dosis no fue capaz de limitar la infestación de las raíces por el nemátodo, fue efectiva para reducir su reproducción, resultando más efectiva que la

Tabla 5. Segundo racimo: rendimiento total y por categorías comerciales $\left(\mathrm{kg} \mathrm{m}^{-2}\right)$ en tomate 'Elpida' tratado al trasplante con diferentes hormonas vegetales y dosis, cultivado en suelo infestado naturalmente con $N$. aberrans.

Table 5. Second fruit truss: total and grade yield $\left(\mathrm{kg} \mathrm{m}^{-2}\right)$ of 'Elpida' tomato treated at transplant with different phytohormones and doses, grown in soil with natural infestation of N. aberrans.

\begin{tabular}{lcccc}
\hline \multicolumn{1}{c}{ Tratamientos } & \multicolumn{3}{c}{ Categorías comerciales } & \multirow{2}{*}{ Total } \\
\cline { 2 - 3 } & Primera & Segunda & Tercera & \\
\hline Ácido salicílico $0,5 \times 10^{-4} \mathrm{M}$ & $0,71 \mathrm{a}$ & $0,45 \mathrm{a}$ & $0,03 \mathrm{a}$ & $1,20 \mathrm{a}$ \\
Ácido salicílico $1,0 \times 10^{-4} \mathrm{M}$ & $1,70 \mathrm{~b}$ & $1,01 \mathrm{a}$ & $0,20 \mathrm{a}$ & $2,92 \mathrm{a}$ \\
Etileno $0,35 \times 10^{-3} \mathrm{M}$ & $1,08 \mathrm{ab}$ & $0,51 \mathrm{a}$ & $0,12 \mathrm{a}$ & $1,72 \mathrm{a}$ \\
Etileno $0,70 \times 10^{-3} \mathrm{M}$ & $1,53 \mathrm{ab}$ & $0,38 \mathrm{a}$ & $0,06 \mathrm{a}$ & $1,97 \mathrm{a}$ \\
Ácido jasmónico $1,00 \times 10^{-4} \mathrm{M}$ & $1,24 \mathrm{ab}$ & $0,50 \mathrm{a}$ & $0,00 \mathrm{a}$ & $1,74 \mathrm{a}$ \\
Ácido jasmónico $1,00 \times 10^{-5} \mathrm{M}$ & $1,50 \mathrm{ab}$ & $0,39 \mathrm{a}$ & $0,08 \mathrm{a}$ & $1,97 \mathrm{a}$ \\
Testigo & $0,63 \mathrm{a}$ & $0,35 \mathrm{a}$ & $0,04 \mathrm{a}$ & $1,02 \mathrm{a}$ \\
$\mathrm{CV}$ & 5,91 & 9,49 & 13,96 & 4,83 \\
$\mathrm{R}^{2}$ & 0,63 & 0,38 & 0,13 & 0,37 \\
$\mathrm{p}$ & 0,0222 & 0,9277 & 0,9147 & 0,2791 \\
\hline
\end{tabular}

Letras distintas en la columna indican diferencias significativas, prueba de Tukey $(\mathrm{p}<0,05)$

Tabla 6. Tercer racimo: rendimiento total y por categorías comerciales $\left(\mathrm{kg} \mathrm{m}^{-2}\right)$ en tomate 'Elpida' tratado al trasplante con diferentes hormonas vegetales y dosis, cultivado en suelo infestado naturalmente con $N$. aberrans.

Table 6. Third fruit truss: total and grade yield $\left(\mathrm{kg} \mathrm{m}^{-2}\right)$ of 'Elpida' tomato treated at transplant with different phytohormones and doses, grown in soil with natural infestation of $N$. aberrans.

\begin{tabular}{lcccc}
\hline \multirow{2}{*}{ Tratamientos } & \multicolumn{3}{c}{ Categorías comerciales } & \multirow{2}{*}{ Total } \\
\cline { 2 - 3 } & Primera & Segunda & Tercera & \\
\hline Ácido salicílico $0,5 \times 10^{-4} \mathrm{M}$ & $1,20 \mathrm{a}$ & $0,35 \mathrm{a}$ & $0,04 \mathrm{a}$ & $1,59 \mathrm{a}$ \\
Ácido salić́lico $1,0 \times 10^{-4} \mathrm{M}$ & $2,20 \mathrm{a}$ & $0,63 \mathrm{a}$ & $0,04 \mathrm{a}$ & $2,86 \mathrm{a}$ \\
Etileno $0,35 \times 10^{-3} \mathrm{M}$ & $1,48 \mathrm{a}$ & $0,43 \mathrm{a}$ & $0,18 \mathrm{a}$ & $2,04 \mathrm{a}$ \\
Etileno $0,70 \times 10^{-3} \mathrm{M}$ & $1,40 \mathrm{a}$ & $0,44 \mathrm{a}$ & $0,14 \mathrm{a}$ & $1,98 \mathrm{a}$ \\
Ácido jasmónico $1,00 \times 10^{-4} \mathrm{M}$ & $1,35 \mathrm{a}$ & $0,16 \mathrm{a}$ & $0,06 \mathrm{a}$ & $1,58 \mathrm{a}$ \\
Ácido jasmónico $1,00 \times 10^{-5} \mathrm{M}$ & $2,10 \mathrm{a}$ & $0,29 \mathrm{a}$ & $0,00 \mathrm{a}$ & $2,39 \mathrm{a}$ \\
Testigo & $0,50 \mathrm{a}$ & $0,22 \mathrm{a}$ & $0,23 \mathrm{a}$ & $0,94 \mathrm{a}$ \\
$\mathrm{CV}$ & 8,83 & 11,90 & 8,32 & 6,49 \\
$\mathrm{R}^{2}$ & 0,53 & 0,25 & 0,86 & 0,53 \\
$\mathrm{p}$ & 0,0540 & 0,5883 & 0,1350 & 0,0509 \\
\hline
\end{tabular}

Letras distintas en la columna indican diferencias significativas, prueba de Tukey $(\mathrm{p}<0,05)$ 
Tabla 7. Cuarto racimo: rendimiento total y por categorías comerciales $\left(\mathrm{kg} \mathrm{m}^{-2}\right)$ en tomate 'Elpida' tratado al trasplante con diferentes hormonas vegetales y dosis, cultivado en suelo infestado naturalmente con $N$. aberrans.

Table 7. Fourth fruit truss: total and grade yield $\left(\mathrm{kg} \mathrm{m}^{-2}\right)$ of 'Elpida' tomato treated at transplant with different phytohormones and doses, grown in soil with natural infestation of $N$. aberrans.

\begin{tabular}{llccl}
\hline \multirow{2}{*}{ Tratamientos } & \multicolumn{3}{c}{ Categorías comerciales } & \multirow{2}{*}{ Total } \\
\cline { 2 - 3 } & Primera & Segunda & Tercera & \\
\hline Ácido salicílico $0,5 \times 10^{-4} \mathrm{M}$ & $1,42 \mathrm{ab}$ & $0,06 \mathrm{a}$ & $0,07 \mathrm{a}$ & $1,56 \mathrm{ab}$ \\
Ácido salicílico $1,0 \times 10^{-4} \mathrm{M}$ & $2,80 \mathrm{~b}$ & $0,94 \mathrm{a}$ & $0,02 \mathrm{a}$ & $3,77 \mathrm{~b}$ \\
Etileno $0,35 \times 10^{-3} \mathrm{M}$ & $1,75 \mathrm{ab}$ & $0,26 \mathrm{a}$ & $0,14 \mathrm{a}$ & $2,15 \mathrm{ab}$ \\
Etileno $0,70 \times 10^{-3} \mathrm{M}$ & $1,46 \mathrm{ab}$ & $0,47 \mathrm{a}$ & $0,00 \mathrm{a}$ & $1,93 \mathrm{ab}$ \\
Ácido jasmónico $1,00 \times 10^{-4} \mathrm{M}$ & $0,50 \mathrm{a}$ & $0,14 \mathrm{a}$ & $0,11 \mathrm{a}$ & $0,75 \mathrm{a}$ \\
Ácido jasmónico $1,00 \times 10^{-5} \mathrm{M}$ & $1,32 \mathrm{ab}$ & $0,27 \mathrm{a}$ & $0,06 \mathrm{a}$ & $1,66 \mathrm{ab}$ \\
Testigo & $0,67 \mathrm{ab}$ & $0,31 \mathrm{a}$ & $0,02 \mathrm{a}$ & $0,99 \mathrm{ab}$ \\
$\mathrm{CV}$ & 9,61 & 14,14 & 20,33 & 9,36 \\
$\mathrm{R}^{2}$ & 0,54 & 0,52 & 1,00 & 0,50 \\
$\mathrm{p}$ & 0,0231 & 0,4064 & 0,7404 & 0,0450 \\
\hline
\end{tabular}

Letras distintas en la columna indican diferencias significativas, prueba de Tukey $(p<0,05)$

Tabla 8. Quinto racimo: rendimiento total y por categorías comerciales $\left(\mathrm{kg} \mathrm{m}^{-2}\right)$ Table 8. Fifth fruit truss: total and grade yield $\left(\mathrm{kg} \mathrm{m}^{-2}\right)$

\begin{tabular}{llccl}
\hline \multirow{2}{*}{ Tratamientos } & \multicolumn{2}{c}{ Categorías comerciales } & \multirow{2}{*}{ Total } \\
\cline { 2 - 3 } & Primera & Segunda & Tercera & \\
\hline Ácido salicílico $0,5 \times 10^{-4} \mathrm{M}$ & $1,58 \mathrm{ab}$ & $0,46 \mathrm{a}$ & $0,03 \mathrm{a}$ & $2,04 \mathrm{bc}$ \\
Ácido salicílico $1,0 \times 10^{-4} \mathrm{M}$ & $2,33 \mathrm{~b}$ & $0,56 \mathrm{a}$ & $0,00 \mathrm{a}$ & $2,97 \mathrm{c}$ \\
Etileno $0,35 \times 10^{-3} \mathrm{M}$ & $1,05 \mathrm{ab}$ & $0,23 \mathrm{a}$ & $0,02 \mathrm{a}$ & $1,31 \mathrm{ab}$ \\
Etileno $0,70 \times 10^{-3} \mathrm{M}$ & $1,89 \mathrm{ab}$ & $0,21 \mathrm{a}$ & $0,02 \mathrm{a}$ & $2,13 \mathrm{bc}$ \\
Ácido jasmónico $1,00 \times 10^{-4} \mathrm{M}$ & $0,58 \mathrm{a}$ & $0,47 \mathrm{a}$ & $0,11 \mathrm{a}$ & $1,17 \mathrm{ab}$ \\
Ácido jasmónico $1,00 \times 10^{-5} \mathrm{M}$ & $1,60 \mathrm{ab}$ & $0,27 \mathrm{a}$ & $0,06 \mathrm{a}$ & $1,69 \mathrm{bc}$ \\
Testigo & $0,47 \mathrm{a}$ & $0,05 \mathrm{a}$ & $0,08 \mathrm{a}$ & $0,61 \mathrm{a}$ \\
$\mathrm{CV}$ & 8,81 & 14,67 & & 4,82 \\
$\mathrm{R}^{2}$ & 0,67 & 0,64 & & 0,85 \\
$\mathrm{p}$ & 0,0062 & 0,2797 & & 0,0001 \\
\hline
\end{tabular}

Letras distintas en la columna indican diferencias significativas, prueba de Tukey $(p<0,05)$

dosis más baja del mismo elicitor. Al mismo tiempo, permitió un adecuado desempeño de la planta, sin implicar un costo por desvío de recursos y energía metabólica que afectaran al crecimiento y reproducción, como fue observado en otras especies (Peteira Delgado-Oramas, 2020).

Los índices de agallamiento y reproducción son criterios válidos para evaluar el grado de resistencia al ataque de Meloidogyne spp. (Cap et al., 1993; Yaghoobi et al., 1995; Bourne y Kerry, 1999; Cortada et al, 2008; Pinheiro et al., 2019). Cortada et al. (2008), evaluando la resistencia genética a Meloidogyne spp. en distintos portainjertos y pies francos de tomate, consideraron la existencia de resistencia cuando el índice de reproducción de un cultivar se diferenciaba significativamente del de un cultivar susceptible, observándose en las condiciones de este estudio un valor significativamente más elevado en las plantas que no fueron tratadas con ninguna de las tres fitohormonas. Asimismo, un menor índice de agallamiento se relaciona con una mayor tolerancia a $N$. aberrans, según fue considerado por Leyva-Mir et al. (2013) al comparar el comportamiento de líneas de tomate frente a patógenos del suelo.

Los elicitores ensayados mostraron, en general, potencial para ser utilizados ante la presencia de 
Tabla 9. Sexto racimo: rendimiento total y por categorías comerciales $\left(\mathrm{kg} \mathrm{m}^{-2}\right)$

Table 9. Sixth fruit truss: total and grade yield $\left(\mathrm{kg} \mathrm{m}^{-2}\right)$

\begin{tabular}{lcccc}
\hline \multirow{2}{*}{ Tratamientos } & \multicolumn{3}{c}{ Categorías comerciales } & \multirow{2}{*}{ Total } \\
\cline { 2 - 3 } & Primera & Segunda & Tercera & \\
\hline Ácido salicílico $0,5 \times 10^{-4} \mathrm{M}$ & $0,47 \mathrm{a}$ & $0,25 \mathrm{a}$ & $0,00 \mathrm{a}$ & $0,72 \mathrm{a}$ \\
Ácido salićlico $1,0 \times 10^{-4} \mathrm{M}$ & $1,78 \mathrm{~b}$ & $0,18 \mathrm{a}$ & $0,08 \mathrm{a}$ & $2,05 \mathrm{~b}$ \\
Etileno $0,35 \times 10^{-3} \mathrm{M}$ & $0,6 \mathrm{a}$ & $0,18 \mathrm{a}$ & $0,02 \mathrm{a}$ & $0,84 \mathrm{ab}$ \\
Etileno $0,70 \times 10^{-3} \mathrm{M}$ & $1,09 \mathrm{ab}$ & $0,36 \mathrm{a}$ & $0,04 \mathrm{a}$ & $1,50 \mathrm{ab}$ \\
Ácido jasmónico $1,00 \times 10^{-4} \mathrm{M}$ & $0,50 \mathrm{a}$ & $0,09 \mathrm{a}$ & $0,15 \mathrm{a}$ & $0,74 \mathrm{a}$ \\
Ácido jasmónico $1,00 \times 10^{-5} \mathrm{M}$ & $1,00 \mathrm{ab}$ & $0,07 \mathrm{a}$ & $0,08 \mathrm{a}$ & $1,15 \mathrm{ab}$ \\
Testigo & $0,62 \mathrm{a}$ & $0,03 \mathrm{a}$ & $0,11 \mathrm{a}$ & $0,76 \mathrm{ab}$ \\
$\mathrm{CV}$ & 6,43 & 8,04 & 7,94 & 6,93 \\
$\mathrm{R}^{2}$ & 0,66 & 0,84 & 1,00 & 0,57 \\
$\mathrm{p}$ & 0,0017 & 0,5685 & 0,6466 & 0,0128 \\
\hline
\end{tabular}

Letras distintas en la columna indican diferencias significativas, prueba de Tukey $(\mathrm{p}<0,05)$

Tabla 10. Características de raíces de tomate 'Elpida' a la finalización del ensayo según tipo y dosis de fitohormona aplicada al trasplante.

Table 10. Characteristics of 'Elpida' tomato roots at the end of the trial according to the kind and dose of phytohormon treatment applied at transplant.

\begin{tabular}{lcccc}
\hline \multicolumn{1}{c}{ Tratamientos } & $\begin{array}{c}\text { Peso fresco } \\
\text { raíz (g) }\end{array}$ & $\begin{array}{c}\text { No de } \\
\text { agallas }\end{array}$ & $\begin{array}{c}\text { Índice de } \\
\text { agallamiento }\end{array}$ & $\begin{array}{c}\text { Índice de } \\
\text { reproducción }\end{array}$ \\
\hline Ácido salicílico $0,5 \times 10^{-4} \mathrm{M}$ & $18,25 \mathrm{a}$ & $36,00 \mathrm{~b}$ & $1,63 \mathrm{~b}$ & $0,06 \mathrm{a}$ \\
Ácido salicílico $1,0 \times 10^{-4} \mathrm{M}$ & $29,50 \mathrm{~b}$ & $1,25 \mathrm{a}$ & $0,00 \mathrm{a}$ & $0,00 \mathrm{a}$ \\
Etileno $0,35 \times 10^{-3} \mathrm{M}$ & $19,75 \mathrm{a}$ & $36,50 \mathrm{~b}$ & $1,68 \mathrm{~b}$ & $0,09 \mathrm{a}$ \\
Etileno $0,70 \times 10^{-3} \mathrm{M}$ & $20,25 \mathrm{a}$ & $49,00 \mathrm{~b}$ & $2,23 \mathrm{~b}$ & $0,12 \mathrm{a}$ \\
Ácido jasmónico $1,00 \times 10^{-4} \mathrm{M}$ & $19,50 \mathrm{a}$ & $55,25 \mathrm{~b}$ & $2,38 \mathrm{~b}$ & $0,16 \mathrm{a}$ \\
Ácido jasmónico $1,00 \times 10^{-5} \mathrm{M}$ & $21,00 \mathrm{a}$ & $42,00 \mathrm{~b}$ & $2,60 \mathrm{~b}$ & $0,06 \mathrm{a}$ \\
Testigo & $21,75 \mathrm{a}$ & $97,50 \mathrm{c}$ & $5,00 \mathrm{c}$ & $5,05 \mathrm{~b}$ \\
\hline
\end{tabular}

Letras distintas en la columna indican diferencias significativas, prueba de Tukey $(p<0,05)$

N. aberrans en el suelo de cultivo, considerando que en la evaluación de distintos nematicidas para el control del mismo nemátodo $(33,6+/-4,8$ $\mathrm{J} 2$ cada $100 \mathrm{~cm}^{3}$ de suelo), la mejor respuesta fue obtenida con la aplicación de dicloropropeno más cloropicrina, lográndose una tasa de reproducción de 0,76 e índices de agallamiento de 1,67 (Cabrera-Hidalgo et al., 2015). En base a los resultados observados, el uso de estos elicitores puede considerarse una estrategia apropiada para el manejo de adversidades, siendo una alternativa benéfica para el medio ambiente y accesible a sistemas de producción convencional (Espinosa Vázquez, 2017); aunque es importante profundizar en la comprensión de los mecanismos de acción, costos biológicos de la activación de las resistencias, y estudios de interacción multitrófica; así como respetar criterios de seguridad toxicológica y ambiental
(Peteira , 2020; Peteira Delgado-Oramas et al., 2020).

La aplicación de ácido salicílico a plantines de tomate susceptibles a Meloidogyne spp. resultó efectiva para reducir el índice de reproducción, cuando se utilizó por inmersión de la raíz por toda una noche $(0,1-1 \mathrm{mM})$ o por mojado del suelo (45 mg L $\mathrm{mg}^{-1}$ ), modo de tratamiento equivalente al usado en este trabajo (Molinari, 2008; Molinari et al., 2014).

La respuesta diferencial al tipo de fitohormona puede estar dada por el hecho de que el ácido salicílico participa en mecanismos de resistencia sistémica adquirida, generando reacciones que perduran en el tiempo y protegen a la planta, independientemente de la etiología del patógeno; mientras que el ácido jasmónico y el etileno actúan como señales para la resistencia sistémica inducida, donde frente al reconocimiento 
del ataque, la interacción entre el patógeno y la especie vegetal es específica e invariable (Camarena-Gutiérrez y de la Torre-Almaráz, 2007; Samaniego-Gámez et al., 2017). Por otra parte, el etileno es generado por las plantas como una primera reacción defensiva al ataque inicial del nemátodo $\mathrm{u}$ otros patógenos, en el momento en que produce la primera lesión celular, detectándose aún antes que la inducción producida por el ácido jasmónico y actuando como precursor en la formación de otros elicitores (Fudali et al., 2013; Laredo et al., 2017; Marhavý et al., 2019). Este modo de acción podría estar produciendo una demora en la activación de las defensas, lo que explicaría el mayor daño observado en la raíz, en comparación a las de las plantas tratadas con la dosis más alta de ácido salicílico. Además, al igual que el ácido jasmónico, se asocia a la defensa ante el ataque de patógenos necrotróficos que producen la muerte de las células desde el principio de la infección para la obtención de nutrientes; mientras que el ácido salicílico participa en el sistema de defensa de las plantas contra el ataque por patógenos biotróficos (Sánchez et al., 2010). En N. aberrans, desde el comienzo de la infestación, las células del parénquima cortical sufren alteraciones fisiológicas y se transforman en una estructura denomina síncito (sitios de alimentación de las hembras), en principio constituido por células con alta actividad metabólica (Tipo A), que deja de ser funcional solo una vez que la hembra muere, quedando en ese momento constituido por un gran número de células muertas (Tipo D) (Doucet et al., 1997; Doucet y Lax, 2005). De esta manera, es esperable una reacción más temprana del sistema de defensas activado por el ácido salicílico, además de una protección más generalizada, que la provocada por el ácido jasmónico o el etileno.

\section{CONCLUSIONES}

En el sitio de ensayo se verificó la presencia de Nacobbus aberrans, en estadios J3 y J4.

En el cultivo previo al implantado para el desarrollo de este estudio, tomate 'Elpida' injertado sobre Maxifort y 9184 presentó en sus raíces una cantidad significativamente menor de huevos y juveniles $\mathrm{J} 2$, respecto a las plantas sin injertar o injertadas sobre Beaufort o Multifort.

En las condiciones del ensayo, el ácido salicílico, ácido jasmónico y etileno redujeron significativamente la infestación de las raíces por $N$. aberrans, promoviendo el incremento del rendimiento respecto a plantas sin tratar.

El ácido salicílico en dosis de $1,00 \times 10^{-4} \mathrm{M}$ se destacó sobre los otros elicitores y dosis, conforme lo demostró el menor número de agallas en las raíces y los nulos índices de agallamiento y reproducción, así como su efecto sobre el rendimiento en el ciclo completo de cultivo y en la producción por racimos.

Los resultados indican el potencial del uso de estos elicitores para reducir los daños provocados por Naccobus aberrans en tomate, siendo de interés continuar investigando las implicancias prácticas de su uso, así como profundizar en la comprensión de su modo de acción sobre este nemátodo.

\section{RECONOCIMIENTOS}

Este trabajo se desarrolló en el marco del Proyecto de Investigación "Ecofisiología de los Cultivos Protegidos" (11/A 321). Programa de Incentivos a la Investigación, UNLP.

\section{LITERATURA CITADA}

Avanci, N., D. Luche, G. Goldman, and M. Goldman. 2010. Jasmonates are phytohormones with multiple functions, including plant defense and reproduction. Genet. Mol. Res. 9:484-505.

Awang, N.A., M.R. Ismail, D. Omar, D., and M.R. Islam. 2015. Comparative study of the application of jasmonic acid and pesticide in chilli: effects on physiological activities, yield and viruses control. Biosci. J. 31(3):672-681.

Azpilicueta, C., M.C. Aruani, y E. Chaves. 2011. Relación entre la nematofauna y la historia del suelo en huertos frutícolas. Agro Sur 39 (1):13-23.

Bourne, J.M., and B.R. Kerry. 1999. Effects of the host plant on the efficacy of Verticillium chlamydosporium as a biological control agent of root-knot nematodes at different densities and fungal applications rates. Soil Biol. Biochem. 31:75-84.

Cabrera-Hidalgo, A.J., E. Valadez Moctezuma, y N. Marbán Mendoza. 2015. Efecto del Fluensulfone sobre la movilidad in vitro, y la reproducción y agallamiento de Nacobbus aberrans en microparcelas. Nematropica 45(1):59-71.

Camarena-Gutiérrez G., y R. de la Torre-Almaráz. 2007. Resistencia sistémica adquirida en plantas: estado actual. Rev. Chapingo ser. Cienc. For. Ambient. 13(2):157-162.

Cap, G.B., P.A. Robert, and L.J. Thomason. 1993. Inheritance of heat-stable resistance to Meloidogyne incognita in Lycopersicon peruvianum and its relationship to the $M i$ gene. Theor. Appl. Genet. 85:777-783. 
Cortada, L., F.J. Sorribasb, C. Ornatb, I. Kaloshianc, and S. Verdejo-Lucas. 2008. Variability in infection and reproduction of Meloidogyne javanica on tomato rootstocks with the Mi resistance gene. Plant Pathology 57:1125-1135.

Coyne, D.L., J.M. Nicol, and B. Claudius-Cole. 2007. Practical plant nematology: a field and laboratory guide. SP-IPM Secretariat, International Institute of Tropical Agriculture (IITA), Cotonou, Benin.

Cristóbal, A.J., I. Cid del Prado V., N. MarbánMendoza, G.P. Sánchez, G. Mora-Aguilera, y L.R.H. Manzanilla. 2001. Sobrevivencia de estadios biológicos de Nacobbus aberrans en condiciones de campo. Nematropica 31(2):227-233.

Di Rienzo J.A., F. Casanoves, M.G. Balzarini, L. Gonzalez, M. Tablada, y C.W. Robledo. 2013. InfoStat versión 2013. Disponible en http:// www.infostat.com.ar (Consulta 5 de marzo de 2015).

Doucet, M., y P. Lax. 2005. El género Nacobbus Thorne \& Alien, 1944 en Argentina. 6. La especie N. aberrans (Thorne, 1935) Thorne \& Alien, 1944 (Nematoda: Tylenchida) y su relación con la agricultura. Academia Nacional de Agronomía y Veterinaria. Buenos Aires, Argentina. Disponible en: http://sedici.unlp.edu.ar/bitstream/ handle/10915/29191/Documento_completo. pdf? sequence $=1 \&$ isAllowed $=y$ (Consulta: 23 de mayo de 2020).

Doucet, M., E. L. De Ponce De León, M.C. Tordable, y N. Poloni. 1997. Nacobbus aberrans y su asociación con vegetales en Argentina. Nematol. Medit. 25:279-285.

Espinosa Vázquez, M.A. 2017. Bacillus subtilis y la fitohormona ácido salicílico, como control del tizón temprano (Alternaria solani) en el cultivo de jitomate (Solanum lycopersicum L.). Tesis de maestro en Ciencias en Manejo Sustentable y Control de Riesgos. Facultad de Ingeniería, Universidad de Ciencias y Artes de Chiapas, Chiapas, México.

Fudali, S.L., C. Wang, and V.M. Williamson. 2013. Ethylene signaling pathway modulates attractiveness of host roots to the root-knot nematode Meloidogyne hapla. MPMI 26(1):7586.

Gheysen, G., and M.G. Mitchum. 2019. Phytoparasitic nematode control of plant hormone pathways. Plant Physiol. 179:12121226.
Hooper, D.J., Hallmann, J., and Subbotin, S. 2005. Methods for extraction, processing and detection of plant and soil nematodes. p. 53-86. In Luc, M., R.A. Sikora, and J. Bridge (eds.). Plant Parasitic Nematodes in Subtropical and Tropical Agriculture. CAB International, Wallingford, U.K.

Laredo, E.I., J.L. Martínez Hernández, A. Iliná, L. Guillen, y F.D. Hernández. 2017. Aplicación de ácido jasmónico como inductor de resistencia vegetal frente a patógenos. Rev. Mex. Cienc. Agríc. 8(3):673-683.

Leyva-Mir, S.G., C.M. González-Solano, J.E. Rodríguez-Pérez, y D. Montalvo-Hernández. 2013. Comportamiento de líneas avanzadas de tomate (Solanum lycopersicum L.) a fitopatógenos en Chapingo, México. Revista Chapingo. Serie Horticultura 19(3):301-313.

López-Martínez, N., M.T. Colinas-León, C.B. Peña-Valdivia, Y. Salinas-Moreno, P. FuentesMontiel, M. Biesaga, and E. Zavaleta-Mejía. 2010. Alterations in peroxidase activity and phenylpropanoid metabolism induced by Nacobbus aberrans Thorne and Allen, 1944 in chilli (Capsicum annuum L.) CM334 resistant to Phytophthora capsici Leo. Plant Soil 338:399409.

Marhavý, P., A. Kurenda, S. Siddique, V. Dénervaud, F. Zhou, J. Holbein, et al. 2019. Single-cell damage elicits regional, nematode-restricting ethylene responses in roots. EMBO J. 38:e100972.

Méndez-Santiago, E.W. 2020. Actividad nematicida de Serratia sp. contra el nemátodo agallador Nacobbus aberrans. Tesis Maestro en Biotecnología. Centro de Investigación en Biotecnología, Universidad Autónoma del Estado de Morelos, Cuernavaca, Morelos, México.

Mitidieri, M.S., M.V. Brambilla, M. Barbieri, E. Arpía, L. Maldonado, R. Celié, et al. 2011. Plantas injertadas sobre pies resistentes: una solución para el cultivo de tomate. p. 61-64. En Mitidieri, M., G. Corbino, y A. Constantino (eds.). Seminario de horticultura urbana y periurbana. Buscamos soluciones entre todos. Ediciones INTA, San Pedro, Argentina.

Molinari, S. 2008. Salicylic acid as an elicitor of resistance to root-knot nematodes in tomato. Acta Hortic. 789:119-126.

Molinari, S., E. Fanelli, and P. Leonetti. 2014. Expression of tomato salicylic acid (SA)responsive pathogenesis-related genes in Mi-1-mediated and SA-induced resistance to root-knot nematodes. Mol. Plant Pathol. 15(3):255-264. 
Pérez-Rodríguez, I. 2008. Control integrado de Nacobbus aberrans en Chile (Capsicum annuum L.) bajo condiciones de invernadero y campo. Tesis Maestro en Ciencias en Fitopatología. Campus Montecillo, Montecillo, Estado de México, México.

Pérez-Rodríguez, I., F. Franco-Navarro, I. Cid del Prado-Vera, and E. Zavaleta-Mejía. 2011. Control of Nacobbus aberrans in chili pepper (Capsicum annuum L.) by the combination of organic amendments, nematophagous fungi and nematicides. Nematropica 41:122-129.

Peteira, B. 2020. La resistencia inducida como alternativa para el manejo de plagas en las plantas de cultivo. Revista de Protección Vegetal 35(1):e07. Disponible en: http:// scielo.sld.cu/scielo.php?script=sci arttext\&pid=S1010-27522020000100001 (Consulta 2 de marzo de 2021).

Peteira, B., I. González, M.G. Rodríguez, y O. Pino. 2020. La resistencia inducida por productos derivados de plantas: alternativa para el manejo de plagas agrícolas. Revista de Protección Vegetal 35(3). Disponible en: http://revistas.censa.edu.cu/index.php/RPV/ article/view/1105 (Consulta 2 de marzo de 2021).

Pinheiro, J.B., G.O. da Silva, V.R. Oliveira, G.B. Amaro, and A.A. Morais. 2019. Prospection of genetic resistance resources to root-knot nematodes in cucurbit genotypes. Hortic. Bras. 37(3):343-347.

Ramirez-Estrada, K., H. Vidal-Limon, D. Hidalgo, E. Moyano, M. Golenioswki, R.M. Cusidó, et al. 2016. Elicitation, an effective strategy for the biotechnological production of bioactive high-added value compounds in plant cell factories. Molecules 21: doi:10.3390/ molecules21020182.

Salas, A., M. Achinelly, E. Chaves, y N. Camino. 2015. Estudio de la diversidad de nematodos asociados al sustrato como indicadores de la calidad del suelo en agroecosistemas. Memorias del V Congreso Latinoamericano de Agroecología. Disponible en: https:// digital.cic.gba.gob.ar/bitstream/ handle/11746/4650/11746_4650.pdf-PDFA. pdf? sequence $=1 \&$ isAllowed $=y$ (Consulta 18 mayo 2020).

Samaniego-Gámez, B.Y., A. Reyes-Ramírez, O.A. Moreno-Valenzuela, y J.M. Tun-Suárez. 2017. Resistencia sistémica inducida contra virus fitopatógenos mediada por la inoculación con la rizobacteria Bacillus spp. Rev. Protección Veg. 32(1):10-22.
Sánchez-Chávez, E., R. Barrera-Tovar, E. MuñozMárquez, D.L. Ojeda-Barrios, y A. AnchondoNájera. 2011. Efecto del ácido salicílico sobre biomasa, actividad fotosintética, contenido nutricional y productividad del chile jalapeño. Revista Chapingo. Serie Horticultura 17:63-68.

Sánchez, G., E. Mercado, D.E. Peña, H. Reyes de la Cruz, y E. Pineda. 2010. El ácido salicílico y su participación en la resistencia a patógenos en plantas. Biológicas 12(2):90- 95.

Suazo Castro, B.R. 2019. Identificación de defensas inducibles a mosca blanca (Trialeurodes vaporariorum) en el cultivo de tomate (Solanum lycopersicum) elicitadas con fitohormonas. Tesis de maestría, Facultad de Ciencias Agrarias y Forestales, Universidad Nacional de La Plata, La Plata, Argentina.

Taylor, A.L., and J.N. Sasser. 1978. Biology, identification and control of rootknot nematodes (Meloidogyne species). Department of Plant Pathology, North Carolina State University, United States Agency for International Development. Raleigh, North Carolina USA.

Thorne, G.D. 1935. The sugar beet nematode and other indigenous nemic parasites of shadscale. J. Agr. Res. 51:509-514.

Thorne, G.D., and M.W. Allen. 1944. Nacobbus dorsalis, nov. gen. nov, spec. (Nematoda: Tylenchidae) producing galls on the roots of alfileria, Erodium cicutarium (L.) L' Her. Proceedings of the Helminthological Society of Washington 11:27-31.

Tucuch-Haas, C., G. Alcántar-González, L.I. Trejo-Téllez, H. Volke-Haller, Y. SalinasMoreno, y A. Larqué-Saavedra. 2017. Efecto del ácido salicílico en el crecimiento, estatus nutrimental y rendimiento en maíz (Zea mays). Agrociencia 51(7):771-781.

Veremis, J.C., G.B. Cap, and P.A. Roberts. 1997. A search for resistance in Lycopersicon spp. to Nacobbus aberrans. Plant Disease 81(2):217222.

Yaghoobi, J., I. Kaloshian, Y. Wen, and V.M. Willamson. 1995. Mapping a new nematode resistance locus in Lycopersicon peruvianum. Theor. Appl. Genet. 91:457-464.

Yildirim, E., and A. Dursun. 2009. Effect of foliar salicylic acid applications on plant growth and yield of tomato under greenhouse conditions. Acta Hortic. 807:395-400.

Zhou J., F. Jia, S. Shao, H. Zhang, G. Li, X. Xia, et al. 2015. Involvement of nitric oxide in the jasmonate-dependent basal defense against root-knot nematode in tomato plants. Front. Plant Sci. 6:193. 